\title{
A Green Method for Graphite Exfoliation, Effect of Milling Intensity.
}

\author{
I. Estrada-Guel ${ }^{1}$, F.C. Robles-Hernandez ${ }^{2}$, C. Carreño-Gallardo ${ }^{1}$ and R. Martínez-Sánchez ${ }^{1}$. \\ 1. Centro de Investigación en Materiales Avanzados (CIMAV). Laboratorio Nacional de Nanotecnología \\ Miguel de Cervantes No. 120, 31109, Chihuahua, Chih., Mexico. \\ .2. Department of Mechanical Engineering Technology, University of Houston, Houston, TX 77204- \\ 4020, USA.
}

Graphite is a natural allotropic form of carbon, being the most stable allotrope under standard conditions; multiple layers or foils named graphitic carbon and graphenes are considered single or double layers of carbon with a honey comb structures that constitute this material. Graphenes have attracted considerable attention of the scientific community, because of their excellent mechanochemical properties, high thermal conductivity and low thermal expansion coefficient [1]. A chemical process to produce single layers graphenes is by inserting ions, atoms or molecules between the layers. During the exfoliation process, elimination of the intercalated species leads to an important material expansion, forming a highly porous material commonly named exfoliated graphite (EG).

Commercial EG exhibits elevated sorption capacity for organics such as heavy oils that can be recovered by absorption. For example, in the literature has been reported that up to $80 \mathrm{~g}$ per gram of heavily oils can be absorbed per gram of EG [2]. This is attributed to the formation of pores [3]. Usually EGs are synthesized by galvanic, chemical and thermal treatments from natural graphite. However, the chemical method is preferred because of its simplicity and versatility. In this method, EG is produced intercalating acid species between the stacked graphene layers with hot $\mathrm{H}_{2} \mathrm{SO}_{4}$ in presence of oxidants such as $\mathrm{KMnO}_{4}, \mathrm{HNO}_{3}$ or $\mathrm{H}_{2} \mathrm{O}_{2}$ [3]. Unfortunately, the reaction remnants are highly corrosive and toxic materials that required careful manipulation; hence, special confinement is required.

In the present work, some EG were prepared using a low cost and environmentally friendly mechanochemical route, which avoids the use of concentrated sulfuric acid and oxidants. Raw materials were: natural graphite, sodium carbonate $\left(\mathrm{Na}_{2} \mathrm{CO}_{3}\right)$ and citric acid that is a weak biodegradable organic acid that is employed as a natural food and drinks conservative. We are using both as defoliation agents. Equiatomic mixtures of $\mathrm{Na}_{2} \mathrm{CO}_{3}$ and natural graphite were milled in a SPEX for 0,1 and $4 \mathrm{~h}$. The milled and raw mixtures were leached with an aqueous citric acid solution ( $8 \% \mathrm{wt} . / \mathrm{wt}$.). Hot refluxed $2 \mathrm{~h}$, washed with distilled water and overnight dried at $80^{\circ} \mathrm{C}$. Morphological and chemical studies of prepared EGs were performed with a high-resolution microscope JSM-7201F.

The Fig. 1 presents some SEM micrographs of graphite particles after the leaching process. It is evident a notable reduction of particle size and an increased level of defoliation as a direct function of milling intensity (time). The adsorption/desorption isotherm curves showed in the Fig. 2 indicate that EG adsorption properties depend of pore characteristics and resulting specific surface area $\left(\mathrm{S}_{\mathrm{sp}}\right)$, both derived of exfoliation degree. In the inner table, it is evident an accentuated Ssp increase in the samples after the leaching process induced by milling.

Common $S_{\mathrm{sp}}$ values reported in the literature are enclosed in the range of 15 [3], 44 [4] to $70 \mathrm{~m}^{2} / \mathrm{g}$ [2]. However, as result of high exfoliation process, the experimental value fund of $123.1 \mathrm{~m}^{2} / \mathrm{g}$, suggests the destruction of the worm-like structure characteristic of EGs [5]. Acknowledgments [6]. 


\section{References:}

[1] T. Kuila, S. Bose, A.K. Mishra, Progress in Materials Science 57 (2012) p. 1061-1105.

[2] B. Tryba, A.W. Morawski, R.J. Kalenczuk, Spill Science \& Technology 8 5-6 (2003) p. 569-571.

[3] M. Inagaki, R. Tashiro, Y. Washino, J. Physics and Chemistry of Solids 65 (2004) p. 133-137.

[4] V. Singh, D. Joung, L. Zhai, Progress in Materials Science 56 (2011) p. 1178-1271.

[5] M. Yang, Y. Hou, N.A. Kotov, Nano Today 7 (2012) p. 430-447.

[6] This research was supported by CONACYT (Project No. 169262) and the Redes Temáticas de

Nanociencias y Nanotecnología (124886).
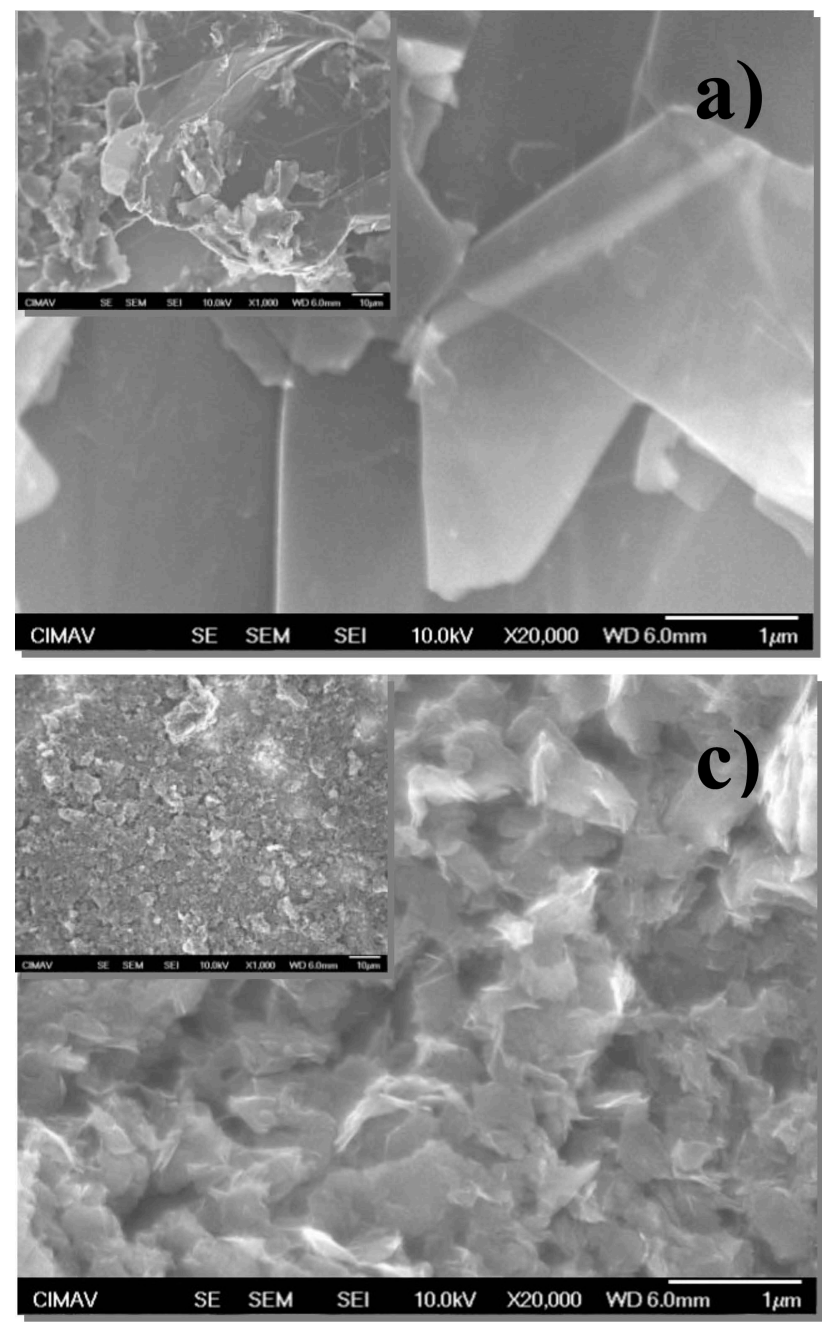

Figure 1. SEM micrographs (1 and $20 \mathrm{KX})$ of graphite particles after the leaching process: a) $0 \mathrm{~h}, \mathrm{~b}$ ) $1 \mathrm{~h}$ and c) $4 \mathrm{~h}$ of milling time.
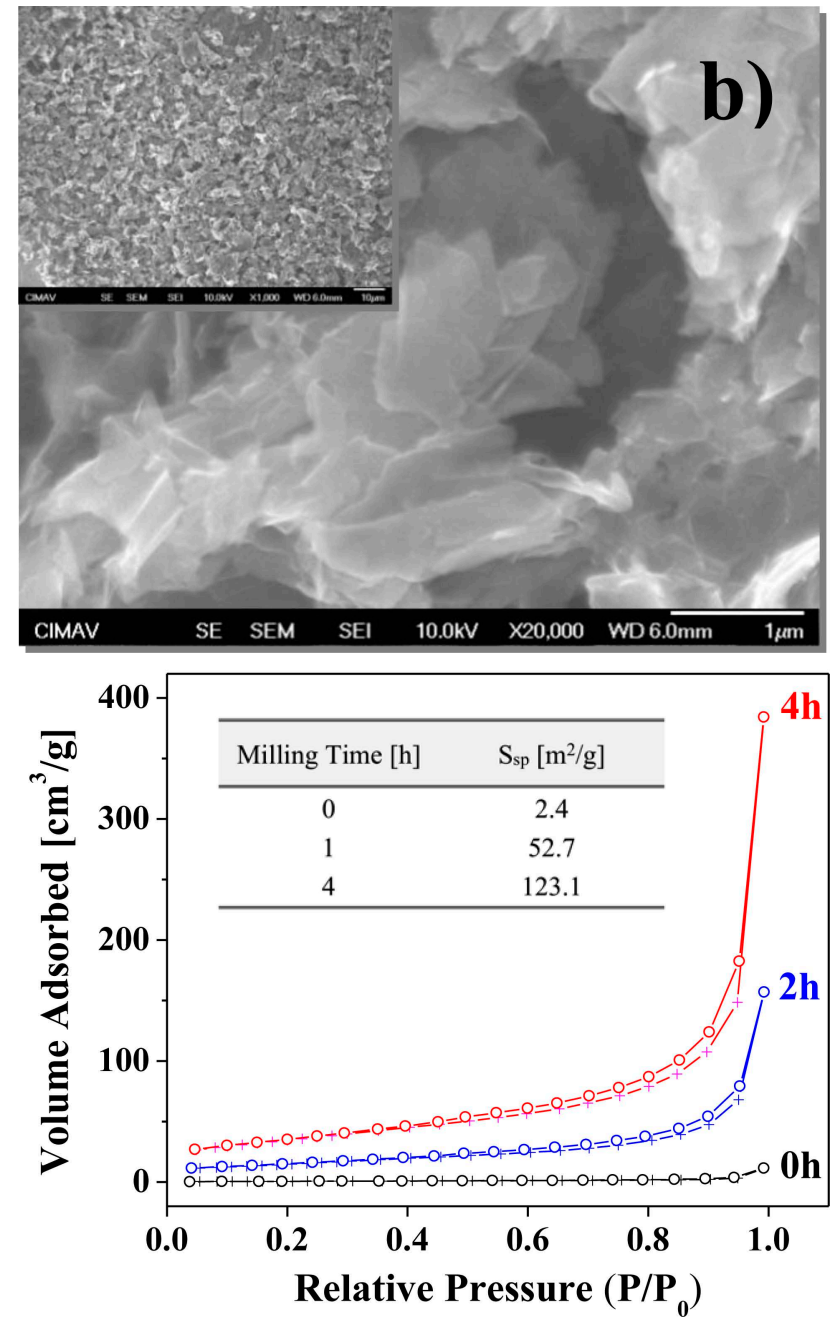

Figure 2. Defoliated graphite isotherm curves are classified into a II type, suggesting the existence of macropores. Low hysteresis between adsorption and desorption isotherms implies a low proportion of mesopores. 